\title{
Effects of Strain Rate Fluctuations on Auto-ignition of Hydrogen/Air Mixture
}

\author{
Gaurav Bansal* and Hong G. $\operatorname{Im}^{\dagger}$ \\ Department of Mechanical Engineering, University of Michigan, Ann Arbor, MI 48109-2125 \\ Su-Ryong Lee \\ Department of Automotive Engineering, Seoul National University of Technology, \\ 172 Gongneung-Dong, Nowon-Gu, Seoul, Korea
}

\begin{abstract}
To understand the auto-ignition behavior in response to the flow turbulence, the effects of scalar dissipation rate fluctuation on the ignition of a nonpremixed hydrogen/air mixture is studied using detailed chemistry in a counterflow configuration. Unsteady scalar dissipation rate is imposed in a sinusoidal form by oscillating the velocity at the nozzle inlet. Mean scalar dissipation rate is chosen such that it is very close to the steady ignition limit, and instantaneous scalar dissipation rate becomes higher than the steady ignition limit for some duration during the induction period. Ignition delay response to frequency of the imposed scalar dissipation rate oscillation is studied for two distinct cases, depending on whether the time average of a cycle of scalar dissipation rate oscillation at ignition kernel is (a) less or (b) greater than the steady ignition limit. For low frequencies, the ignition delay response for both cases is quasi-steady in that it correlates well with the mean scalar dissipation rate. However, at high frequencies the ignition delay response is significantly different for the two cases. For case (a), the ignition delay increases with frequency and levels off at higher frequencies. On the other hand, for case (b) ignition delay increases monotonically with frequency up to a critical value, beyond which no ignition is observed. The high frequency behavior is attributed to the excursion time effect. A newly defined ignition parameter is proposed based on the ignition kernel Damköhler number such that all the unsteady effects of scalar dissipation rate oscillation on ignition delay can be uniquely mapped to this parameter. Subsequently, a new criterion for ignitibility is proposed based on this parameter.
\end{abstract}

\section{Nomenclature}

$\bar{\chi} \quad$ Mean scalar dissipation rate in the induction period, $\mathrm{sec}^{-1}$

$\chi_{s} \quad$ Steady ignition limit, $\mathrm{sec}^{-1}$

$\chi_{a v}$ Time average of a cycle of scalar dissipation rate oscillation, $\mathrm{sec}^{-1}$

$f \quad$ Frequency of velocity fluctuation at the nozzle, $\mathrm{Hz}$

$A \quad$ Amplitude of velocity fluctuation at the nozzle

$\mathrm{t}_{\text {ign }}$ Ignition delay (sec)

$\mathrm{Da}_{\mathrm{H}}$ Ignition kernel Damköhler number, based on $\mathrm{H}$ species

$\Gamma \quad$ Ignition parameter

${ }^{*}$ Graduate Student, AIAA Student Member.

$\dagger$ Associate Professor, AIAA Member.

‡Professor. 


\section{Introduction}

Autoignition of various fuel/air mixtures in a counterflow system has been a subject of extensive studies in the past. It is well understood that autoignition occurs when the rate of radical generation by chemical branching (which may also be coupled with thermal feedback) exceeds the rate of radical loss by transport, where the ratio of the former to the latter is characterized by the Damköhler number. A counterflow system allows a simplified and systematic model configuration in which the characteristic rates of reaction and transport can be independently controlled by adjusting the boundary conditions for composition, temperature, and velocities. In particular, the variation in the boundary velocities, or equivalently in the strain rate, represents the scalar dissipation out of the ignition kernel, and is considered an essential building block in understanding autoignition behavior in turbulent flows. A number of previous studies ${ }^{1-5}$ have reported detailed description of the chemical and thermal structure of ignition kernels and have identified the ignition limits for hydrogen and hydrocarbon fuels under a wide range of the strain rate conditions.

More recently, the effects of unsteady strain rate have also been studied for hydrogen/air ${ }^{6}$ and methane/air mixtures, ${ }^{7}$ considering a monochromatic sinusoidal strain rate oscillation. Sung and Law ${ }^{6}$ found that an initially non-ignitable system may ignite under oscillatory conditions if the excursion time over favorable strain conditions was long enough compared to a characteristic ignition delay time. Consistent results were found by Mason et al. ${ }^{8}$ who studied the effects of impulsive strain rate forcing on ignition of non-premixed hydrogen/air mixtures. In this study, an ignition criterion based on the instantaneous Damköhler number was found to be a good measure in determining the ignitability of the local mixture subjected to a transient conditions.

The above results from unsteady ignition studies may seem to suggest that the ultimate fate of an ignition kernel depends on the cumulative history of the temporal excursion of the strain rate during the steady ignition delay. In our recent study, ${ }^{9}$ however, the effect of temperature fluctuations on the ignition of a homogeneous mixture was found to be two-fold: while the cumulative mean temperature dictates the ignition behavior for low frequency oscillations, as the frequency increases ignition behavior depends more strongly on the instantaneous phasing of the unsteady fluctuation. This result suggests that the ignition behavior in a one-dimensional unsteady system may be more complex than might be expected from the previous studies.

Therefore, in this paper we revisit the effects of sinusoidal fluctuations in the scalar dissipation rate on the autoignition behavior of a nonpremixed hydrogen/air system. This investigation was in part motivated by the recent development of the low temperature combustion (LTC) engines, such as the homogeneous charge compression ignition (HCCI) engines, in which the start of combustion is determined by autoignition of mixture in the presence of velocity and composition fluctuations at varying degrees. Therefore, there is a strong interest in fundamental understanding of the overall effect of turbulence on autoignition for a wide range of parametric conditions. Considering the findings from previous studies, we extend the parametric studies to consider a wider range of frequencies and different mean scalar dissipation rate with respect to the steady ignition limit. Detailed investigation of the ignition kernel growth will be given. Subsequently, a unified ignitability condition is proposed based on the ignition kernel Damköhler number.

\section{Formulation and Numerical Method}

The computational configuration is a standard counterflow mixing layer between two opposing axisymmetric nozzles separated by a fixed distance. The conservation equations for this configuration can be found in Refs., ${ }^{7,10}$ where a compressible formulation was used in order to capture fast transients associated with ignition. The governing equations are solved using OPUS, ${ }^{10}$ which is an opposed-flow solver using a onedimensional similarity coordinate. The code employs variable-order implicit time integration with adaptive time stepping for robust handling of numerical stiffness. ${ }^{11}$ The code is interfaced with Chemkin ${ }^{12}$ and Transport ${ }^{13}$ packages for computing detailed reaction rates and transport properties.

Detailed hydrogen mechanism with 19 reactions and 9 species $^{14}$ is used in this study. For all the results presented in this study, $\mathrm{H}_{2}(50 \%)$ diluted with $\mathrm{N}_{2}(50 \%)$ is flowed from one nozzle against air $\left(\mathrm{N}_{2}-79\right.$ $\%$ and $\mathrm{O}_{2}-21 \%$ ) flowing from the other nozzle $0.5 \mathrm{~cm}$ apart. The pressure is fixed to $2 \mathrm{~atm}$. Fuel side temperature is fixed to $300 \mathrm{~K}$ and air side temperature is fixed to $1020 \mathrm{~K}$. The crossover temperature at this pressure is found to be $985 \mathrm{~K}$, by equating the rates of branching and termination reactions. ${ }^{15}$ The oxidizer temperature, and also the ignition kernel temperature is found to be higher than this, and therefore the 
condition corresponds to that of high temperature ignition chemistry. To study unsteady scalar dissipation rate effects, a time-varying velocity is specified at both the nozzle inlets, as is given by Eq. 1:

$$
V(t)=V_{0}(1+A \sin (2 \pi f t))
$$

where $V_{0}$ is the initial value of velocity, and $A$ and $f$ are the amplitude and frequency of velocity oscillation, respectively. $V_{0}, A$ and $f$ are chosen same for both the nozzles. To compute ignition delay, first a steady computation is performed with reactions suppressed using OPPST, which is a steady version of OPUS. This generates a converged initial solution for the unsteady computation. Then the unsteady computation is performed with reactions turned on at $\mathrm{t}=0$. Although this kind of initial condition is difficult to reproduce in an experimental setup, it is the most feasible initial condition for the present configuration.

\section{Ignition Response to Steady Scalar Dissipation Rate}

First the response of ignition delay to steady scalar dissipation rate is studied. For all the results presented in this study, mixture fraction is defined using the Bilger's ${ }^{16}$ definition as:

$$
\mathrm{Z}=\frac{\mathrm{Y}_{\mathrm{H}} / 2 \mathrm{~W}_{\mathrm{H}}-\left(\mathrm{Y}_{\mathrm{O}}-\mathrm{Y}_{\mathrm{O}, \text { air }}\right) / \mathrm{W}_{\mathrm{O}}}{\mathrm{Y}_{\mathrm{H}, \text { fuel }} / 2 \mathrm{~W}_{\mathrm{H}}+\mathrm{Y}_{\mathrm{O}, \text { air }} / \mathrm{W}_{\mathrm{O}}}
$$

And the scalar dissipation rate is defined as:

$$
\chi=2 \mathrm{D}\left(\frac{\partial \mathrm{Z}}{\partial \mathrm{x}}\right)^{2}
$$

where $\mathrm{D}$ is the thermal diffusivity. Ignition delay is defined as the instant at which rate of change of maximum temperature in the domain becomes maximum. Ignition kernel is identified as the location at which the rate of heat release is maximum.

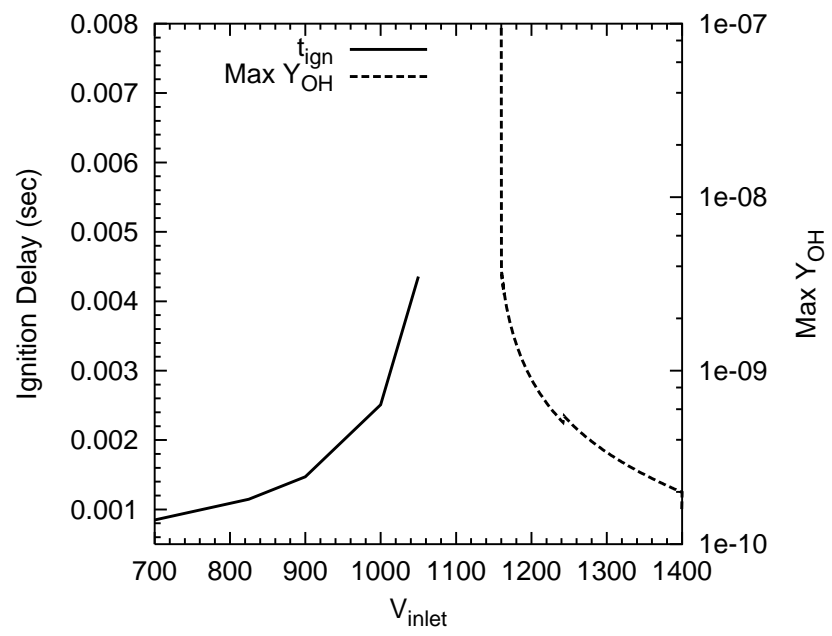

Figure 1. Ignition delay and max $\mathrm{Y}_{\mathrm{OH}}$ as a function of velocity at nozzle inlet

Fig. 1 shows ignition delay plotted against nozzle inlet velocity. As inlet velocity increases, the scalar dissipation rate at ignition kernel increases. This increases the loss of radicals and heat from the ignition kernel and thus a rise in ignition delay occurs. To find the steady ignition limit, a series of steady computations are performed and the inlet velocity is reduced for each computation till ignition turning point occurs. Fig. 1 also shows maximum steady state $\mathrm{OH}$ mass fraction in the domain as a function of inlet velocity. Note that ignition delay plotted on the left axis is obtained from the unsteady computation, whereas maximum $\mathrm{OH}$ mass fraction plotted on the right axis is obtained from a steady computation. From this figure, ignition turning point can be identified to occur at $\mathrm{V}_{\text {inlet }} \approx 1155 \mathrm{~cm} / \mathrm{s}$. If the velocity is greater than this value, no ignition occurs and a frozen solution is obtained. Scalar dissipation rate at the ignition kernel at the steady ignition limit $\left(\chi_{s}\right)$ is found to be $47 \mathrm{~s}^{-1}$. These steady ignition limit results will be used to explain the results for unsteady scalar dissipation rate cases presented in the next section. 


\section{Ignition Response to Unsteady Scalar Dissipation Rate}

Next the response of ignition delay to unsteady scalar dissipation rate is studied. Two cases are selected to study the unsteady scalar dissipation rate effects on ignition. For both cases initial value $\left(V_{0}\right)$ of velocity oscillation at nozzle inlet is fixed to $1050 \mathrm{~cm} / \mathrm{s}$. For case A, amplitude of velocity oscillation at nozzle inlet is fixed to 0.4 , and for case B, amplitude is fixed to 0.8 . Since $V_{0}$ is very close to steady ignition limit, nozzle velocity becomes much greater than steady ignition limit for both cases for some duration in a cycle. The quantity which most directly influences the ignition delay is the scalar dissipation rate at the ignition kernel. Hereafter, the symbol $\chi$ will denote the scalar dissipation rate measured at the ignition kernel, unless stated otherwise. Scalar dissipation rate response for case A and case B are shown in Figs. 2 and 3 where $\chi$ is plotted as a function of time for a few representative frequencies of inlet velocity oscillation.

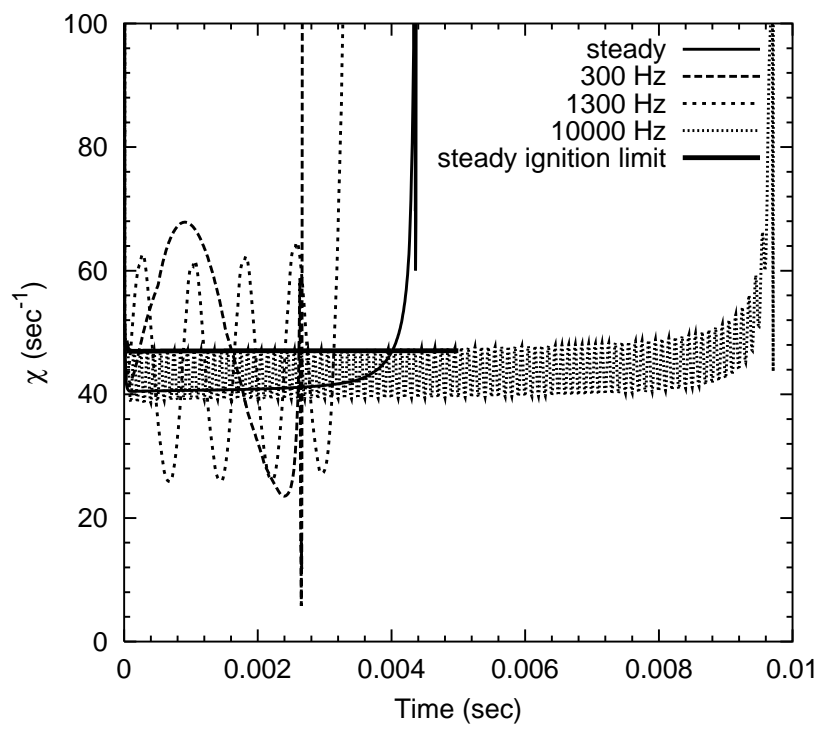

Figure 2. Scalar dissipation rate at ignition kernel as a function of time for a few representative frequencies of inlet velocity oscillation, for case A

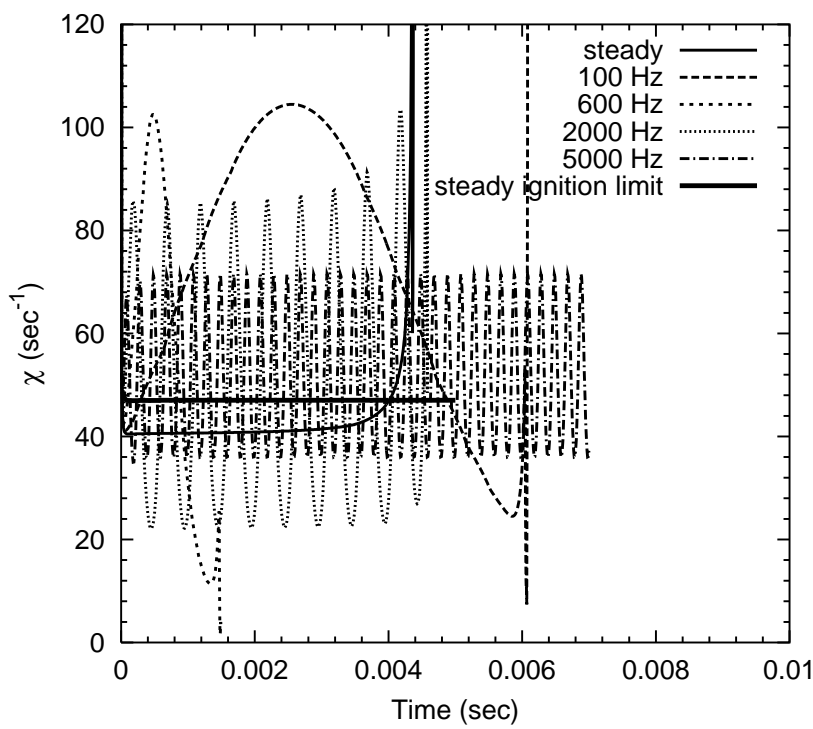

Figure 3. Scalar dissipation rate at ignition kernel as a function of time for a few representative frequencies of inlet velocity oscillation, for case B

We can see that $\chi$ responds sinusoidally for sinusoidal velocity fluctuation at the boundary. Also, as 


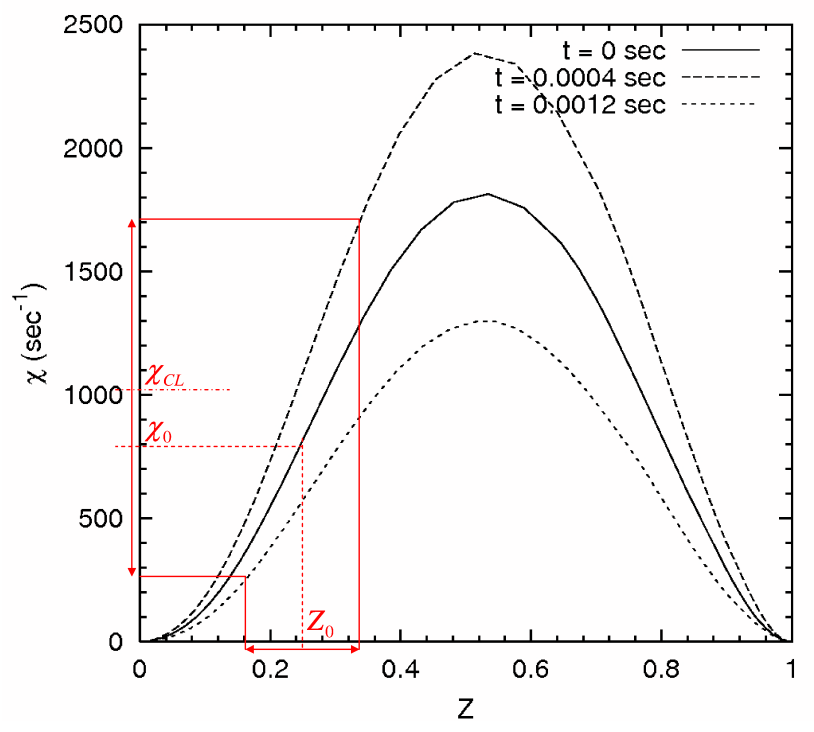

Figure 4. Scalar dissipation rate as a function of mixture fraction at different time instants

one would expect, $\chi$ response gets attenuated as frequency increases. In both these figures, $\chi$ at the steady ignition limit is also plotted. The most important difference which makes cases A and B distinct is that for case $\mathrm{A}$, centerline of scalar dissipation rate oscillation $\left(\chi_{a v}\right)$ is below the steady ignition limit $\left(\chi_{s}\right)$, whereas for case B $\chi_{a v}$ lies above $\chi_{s}$. Although both cases have same $V_{0}$, they donot have the same $\chi_{a v}$. This is because $\chi_{a v}$ for any non-zero frequency does not correspond to the $\chi$ value for the steady case. This is not directly obvious but can be explained as follows. Fig. 4 shows the plots of scalar dissipation rate as a function of mixture fraction at three different time instances. These times correspond to the initial time, the time for velocity crest $(t=0.0004 \mathrm{sec})$ and the time for velocity trough $(t=0.0012)$ at the boundary, for a frequency of $600 \mathrm{~Hz}$. It shows standard mixing layer profile of $\chi$ and $\chi$ is high when velocity at the boundary is high. The initial value of ignition kernel location $\left(\mathrm{Z}_{0}\right)$ and the corresponding $\chi_{0}$ are also shown. Now, during the induction period, mixture fraction at the kernel $\left(Z_{\text {kernel }}\right)$ itself fluctuates. Lets say that $Z_{\text {kernel }}$ fluctuates as shown by the horizontal arrows (on the $\mathrm{x}$-axis). Since, $\chi$ is a function of both the location of ignition kernel $\left(Z_{\text {kernel }}\right)$ (where it is measured) and the velocity at nozzle inlet, it fluctuates as shown by the vertical arrows (on the $\mathrm{y}$-axis). So, it is clear that $\chi_{a v}$ need not be same as $\chi_{0}$. This causes $\chi_{a v}$ to be different for case $\mathrm{A}$ and case $\mathrm{B}$ although both have the same $V_{0}$. Also from Figs. 2 and 3 we note that for all frequencies greater than zero, $\chi_{a v}$ does not depend on frequency. This positioning of $\chi_{a v}$ above or below the steady ignition limit is important because for high frequencies $\chi$ asymptotes towards $\chi_{a v}$, therefore, case A asymptotes towards an ignitable limit and case B asymptotes towards a non-ignitable limit. Thus, one can predict that this would lead to a different high frequency response of ignition delay for the two cases, as is shown next.

Figs. 5 and 6 show the ignition delay response as a function of frequency for cases A and B respectively. Inset of these figures show the zoomed in low frequency response for the two cases. For both cases, ignition delay shows an initial sharp rise and drop as frequency increases. And as expected, the two cases show different behavior for high frequencies. For high frequencies, ignition delay in case A has a sharp rise, which ultimately levels off and becomes constant for very high frequencies. Whereas for case B, ignition delay increases for frequencies upto $2800 \mathrm{~Hz}$, and higher frequencies do not ignite. This leads us to the separation of the frequency range into two regimes: low frequency regime where mean scalar dissipation rate in the induction period governs the ignition response, and high frequency regime where excursion time as defined next governs ignition response.

\section{A. Mean effect Vs Excursion time effect}

To identify the critical frequency which separates the low and the high frequency regimes, ignition delay is plotted as a function of the product of frequency and ignition delay $\left(f^{*} t_{i g n}\right)$ in Fig. 7 . The product $f^{*} t_{\text {ign }}$ 


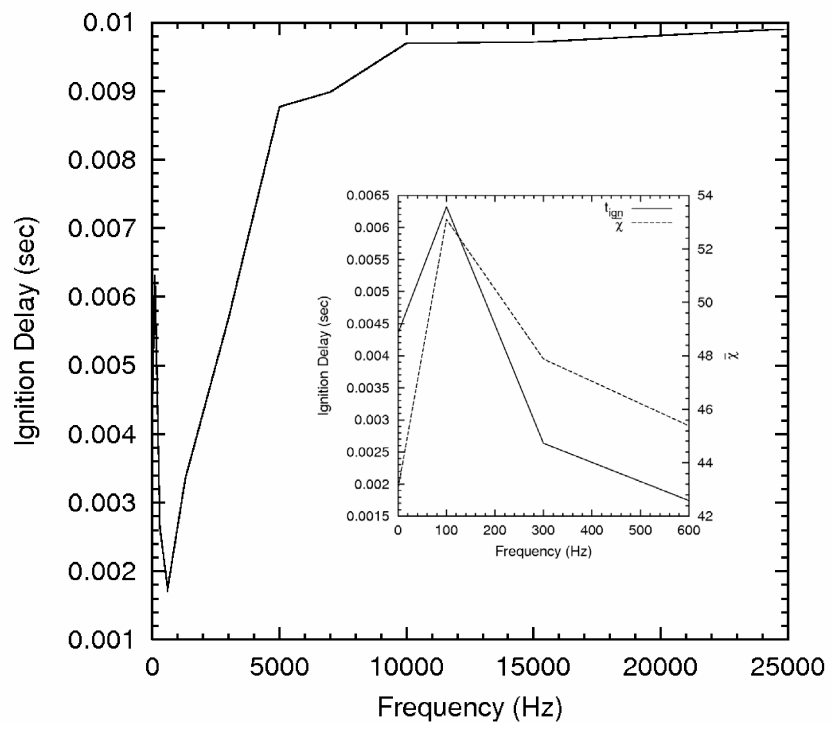

Figure 5. Ignition delay as a function of frequency for case A. Inset: zoomed in low frequency regime, ignition delay and mean scalar dissipation rate as a function of frequency

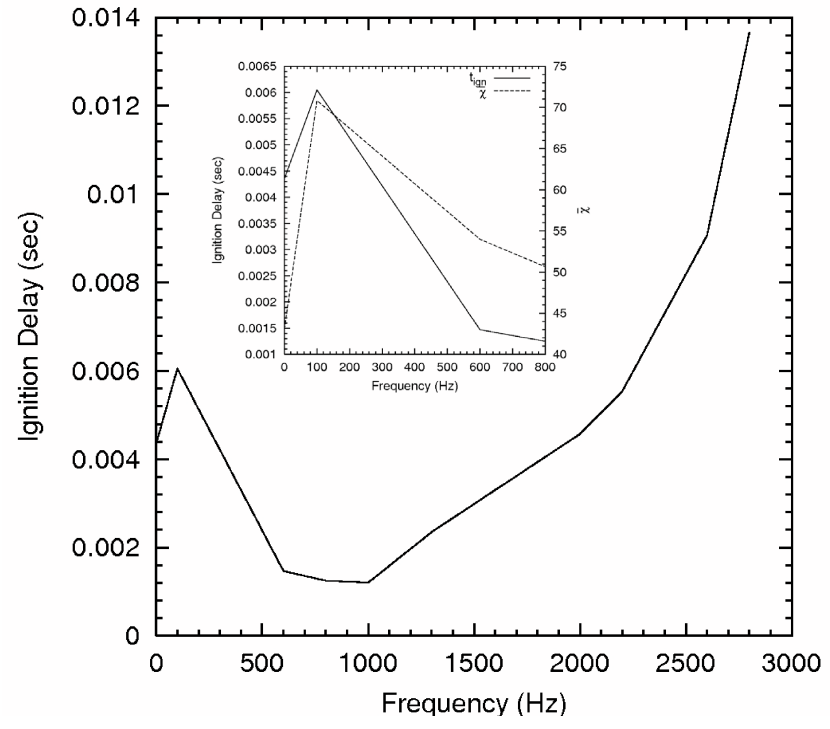

Figure 6. Ignition delay as a function of frequency for case B. Inset: zoomed in low frequency regime, ignition delay and mean scalar dissipation rate as a function of frequency 


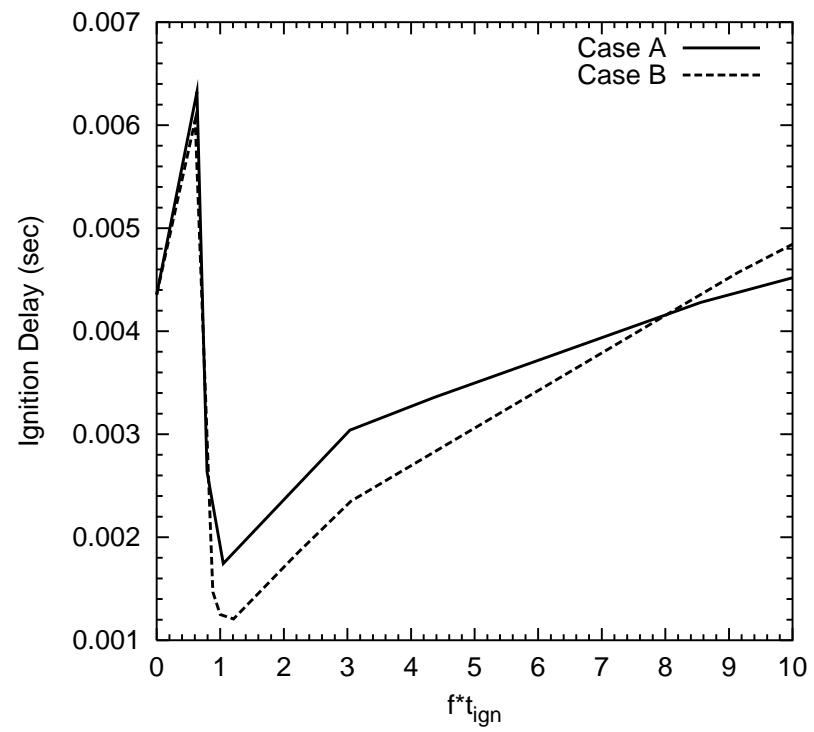

Figure 7. Ignition delay as a function of frequency*ignition delay for cases A and B

gives the number of cycles of $\chi$ oscillation that the ignition kernel goes through before ignition. From the figure it is clear that for both cases ignition delay rises and falls initially upto the point where $\mathrm{f}^{*} \mathrm{t}_{\text {ign }}=1$. After that we see that the ignition delay starts to rise. Thus we separate the frequency range based on the criterion: $\mathrm{f}^{*} \mathrm{t}_{\mathrm{ign}}=1$, or in other words we see "mean" effect when the ignition kernel is exposed to less than one cycle of $\chi$ oscillation, and we see "excursion time" effect when ignition kernel is exposed to more than one cycle of $\chi$ oscillation in the duration of ignition delay. "Mean" effect essentially means that ignition response is quasi steady in the sense that mean scalar dissipation rate $(\bar{\chi})$ at the ignition kernel as defined in Eq. 4 governs the ignition delay. In order to confirm this, $\bar{\chi}$ is plotted as a function of frequency for cases A and B in the inset of Figs. 5 and 6 respectively. We can see that for low frequencies $\bar{\chi}$ correlates well with ignition delay. This quasi-steady behavior of ignition response to low frequencies is in consonance with the results we obtained in our recent work, ${ }^{9}$ in which we studied ignition of homogeneous hydrogen-air mixture subjected to unsteady temperature oscillations. There we found that ignition delay response for low frequencies correlated well with the mean temperature upto ignition delay.

$$
\bar{\chi}=\frac{1}{t_{\text {ign }}} \int_{0}^{t_{i g n}} \chi d t
$$

When the frequency is high enough such that ignition kernel is exposed to more than one cycle of $\chi$ oscillation, hydrogen radical $(\mathrm{H})$ mass fraction goes through excursions of growth and loss at the ignition kernel as $\chi$ oscillates above and below $\chi_{s}$, and ignition event is a cumulative effect of all such cycles. Noteworthy is the point that since the system response is unsteady, an instantaneous $\chi$ value greater than $\chi_{s}$ does not directly correspond to a loss in H mass fraction. Thus to correctly explain the "excursion time" effect we investigate the Damköhler number response, in the next subsection.

\section{B. Damköhler number response to oscillatory $\chi$}

Following the previous study, ${ }^{8}$ the kernel Damköhler number is defined based on the hydrogen atom:

$$
\mathrm{Da}_{\mathrm{H}}=\left|\frac{\dot{\omega}_{\mathrm{H}}}{\rho \mathrm{u}\left(\partial \mathrm{Y}_{\mathrm{H}} / \partial \mathrm{x}\right)+\partial\left(\rho \mathrm{Y}_{\mathrm{H}} \mathrm{V}_{\mathrm{H}}^{\mathrm{D}}\right) / \partial \mathrm{x}}\right|
$$

It is computed at the ignition kernel location and it represents the ratio of gain in $\mathrm{Y}_{\mathrm{H}}$ due to reaction to loss in $Y_{H}$ from the ignition kernel due to convection and diffusion. Figs. 8 and 9 show the transient evolution of $\mathrm{Da}_{\mathrm{H}}$ and $\mathrm{Y}_{\mathrm{H}}$ as reactions occur for a few representative frequencies.

The curves start from bottom right, where $\mathrm{Y}_{\mathrm{H}}$ is very small and $\mathrm{Da} \mathrm{a}_{\mathrm{H}}$ is large. $\mathrm{Da} \mathrm{a}_{\mathrm{H}}$ is large in the beginning because no gradients in $\mathrm{H}$ mass fraction are present and therefore the denominator for Eq. 5 is 


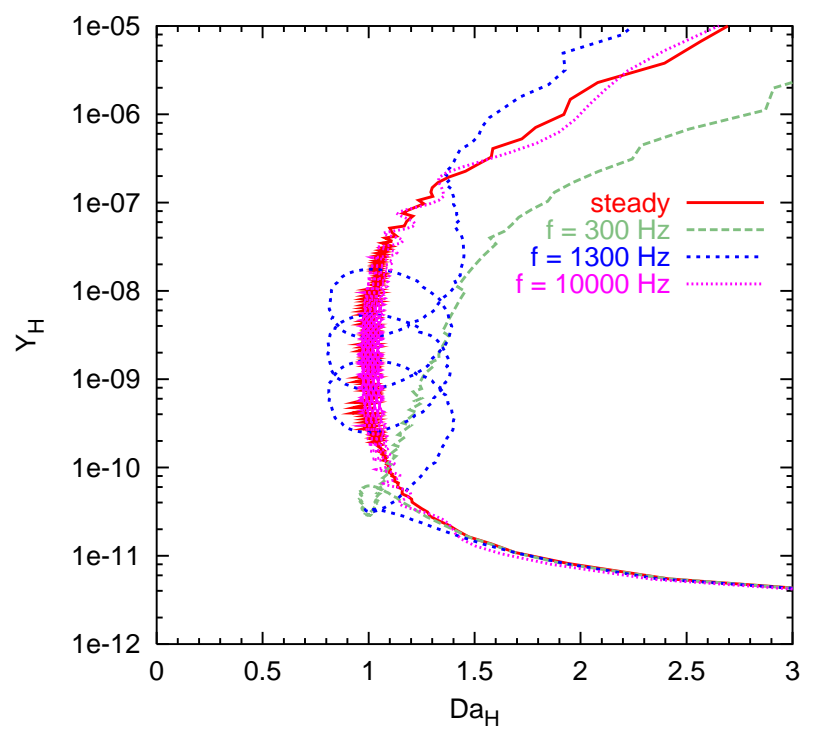

Figure 8. Transient evolution of $\mathrm{Da}_{\mathrm{H}}$ and $\mathrm{Y}_{\mathrm{H}}$ as ignition event takes place, at various frequencies for case $\mathrm{A}$

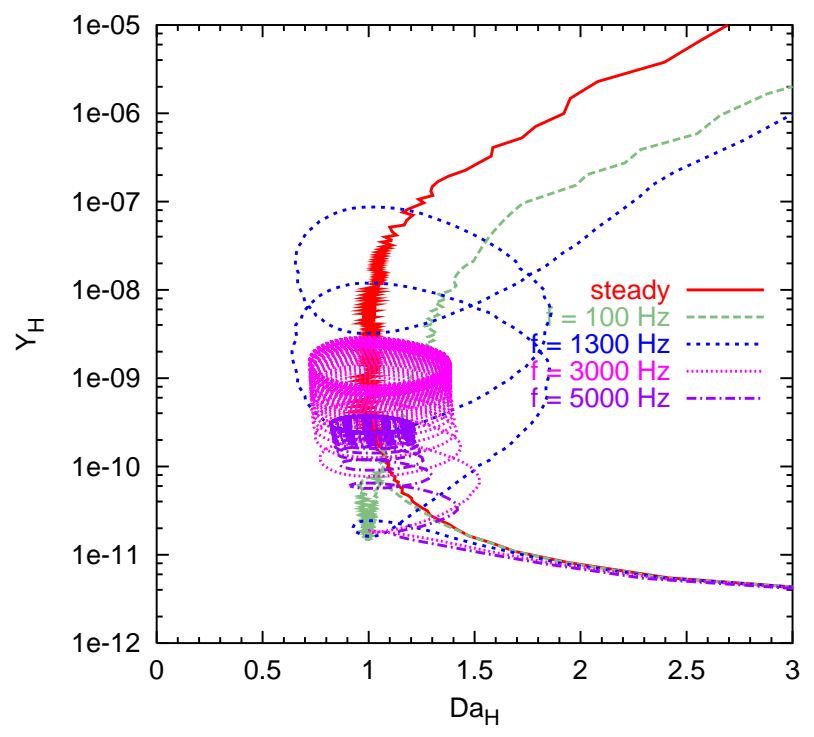

Figure 9. Transient evolution of $\mathrm{Da}_{\mathrm{H}}$ and $\mathrm{Y}_{\mathrm{H}}$ as ignition event takes place, at various frequencies for case $\mathrm{B}$ 
very small. As time increases, $Y_{H}$ starts to increase, and the growth of $Y_{H}$ takes place in a helical pattern because of fluctuations in $\chi$. Also $\mathrm{Da}_{\mathrm{H}}$ fluctuates in response to $\chi$ fluctuations.

Note that $\mathrm{Da}_{\mathrm{H}}$ can become less than one during the induction period and ignition can still occur. That means that there can be a net loss of $\mathrm{H}$ radicals from the kernel for some time during the cycle, and ignition can still occur. We can also see that as frequency increases the centerline of Damkohler number oscillation shifts towards left and becomes more and more close to 1. For case B, plots for high frequencies ( $\mathrm{f}=3000$ $\mathrm{Hz}$ and $\mathrm{f}=5000 \mathrm{~Hz}$ ) for which ignition does not occur, show that a limit cycle is reached for $\mathrm{Y}_{\mathrm{H}}$.

In order to determine the criterion for ignitability and to obtain a unified mapping of ignition delay response to the imposed frequency, we propose a new ignition parameter $(\Gamma)$ as the ratio of time for which $\mathrm{Da}_{\mathrm{H}} \geq 1$ to the ignition delay, as defined in Eq. 6:

$$
\Gamma=\frac{\operatorname{time}\left(\mathrm{Da}_{\mathrm{H}} \geq 1\right)}{t_{\mathrm{ign}}}
$$

In other words, $\Gamma$ represents the fractional duration of favorable condition for ignition. For the cases in which ignition does not occur, we replace the denominator in Eq. 6 by the time for which this ratio converges.

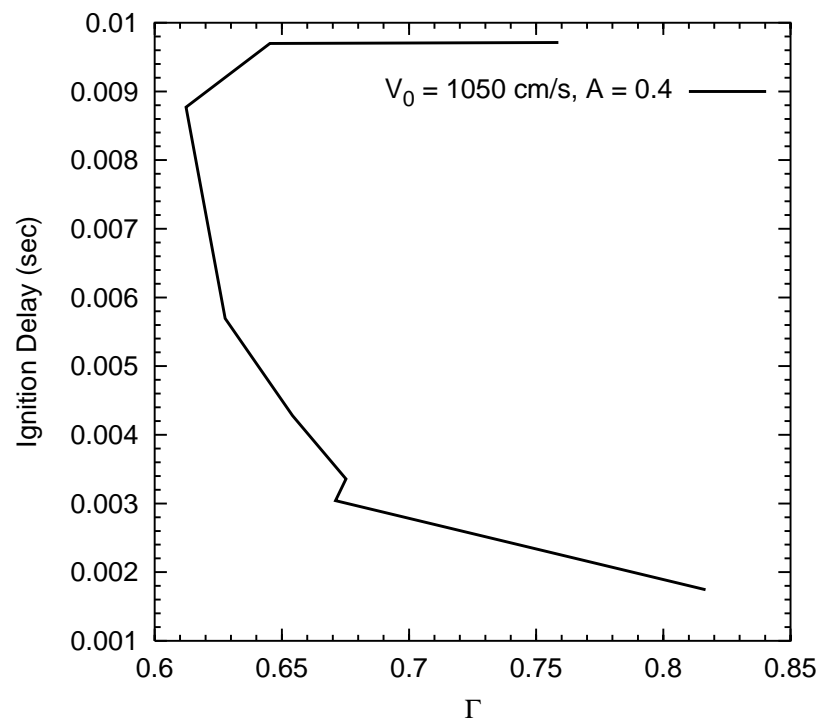

Figure 10. Ignition delay as a function of ignition parameter for case $\mathbf{A}\left(\chi_{a v}<\chi_{s}\right)$

Fig. 10 shows the plot of ignition delay as a function of $\Gamma$ for case $\mathrm{A}\left(\chi_{a v}<\chi_{s}\right)$. Each point in this curve represents a different frequency in the high frequency regime and frequency increases as we go up the curve. The figure shows an increase in ignition delay as $\Gamma$ reduces. This is consistent with the definition of $\Gamma$. However, the reduction in $\Gamma$ as frequency increases is actually counter-intuitive and the reason for this is explained as follows. As frequency increases, the amplitude of $\chi$ oscillation reduces, and hence less and less part of $\chi$ cycle crosses the steady ignition limit since $\chi_{a v}<\chi_{s}$, and therefore one might think that $\Gamma$ should actually increase as frequency increases. However, as mentioned before due to the unsteady nature of the problem, duration for which $\mathrm{Da}_{\mathrm{H}}<1$ in a cycle does not correspond to the duration for which $\chi>\chi_{s}$ and there is a range of frequencies for which $\Gamma$ actually reduces. Ulitmately, for very high frequencies, an asymptotic limit is reached and ignition delay levels off since $\chi$ asymptotes towards $\chi_{a v}$. $\Gamma$ starts to increase for very high frequencies because $\chi$ amplitude attenuates more and more as frequency increases and very less part of $\chi$ cycle lies above $\chi_{s}$.

Next, we plot ignition delay as a function of $\Gamma$ for case $\mathrm{B}\left(\chi_{a v}>\chi_{s}\right)$ in Fig. 11. Three more cases with different values of initial velocity and amplitude $\left[V_{0}=1050, A=-0.8\right],\left[V_{0}=1050, A=0.7\right]$ and $\left[V_{0}=1100\right.$, $A=0.7]$ are included. The negative amplitude means that the phase of velocity oscillation is shifted by 180 degrees. All of these cases also have $\chi_{a v}>\chi_{s}$. Each point on these curves again represent a different frequency in the high frequency regime and frequency increases as we go up the curves. We can see that as $\Gamma$ reduces, ignition delay rises. In this case the duration for which $\chi>\chi_{s}$ correlates well with duration for $\mathrm{Da}_{\mathrm{H}}<1$ in a $\chi$ cycle. As frequency increases amplitude of $\chi$ oscillation attenuates and thus less part 


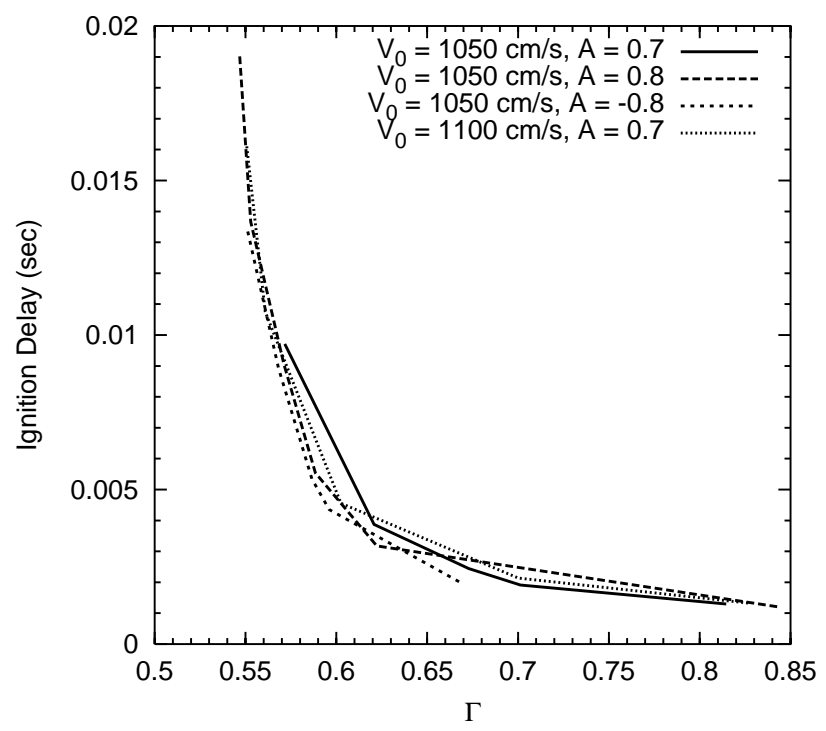

Figure 11. Ignition delay as a function of ignition parameter for case B and other cases with $\chi_{a v}>\chi_{s}$

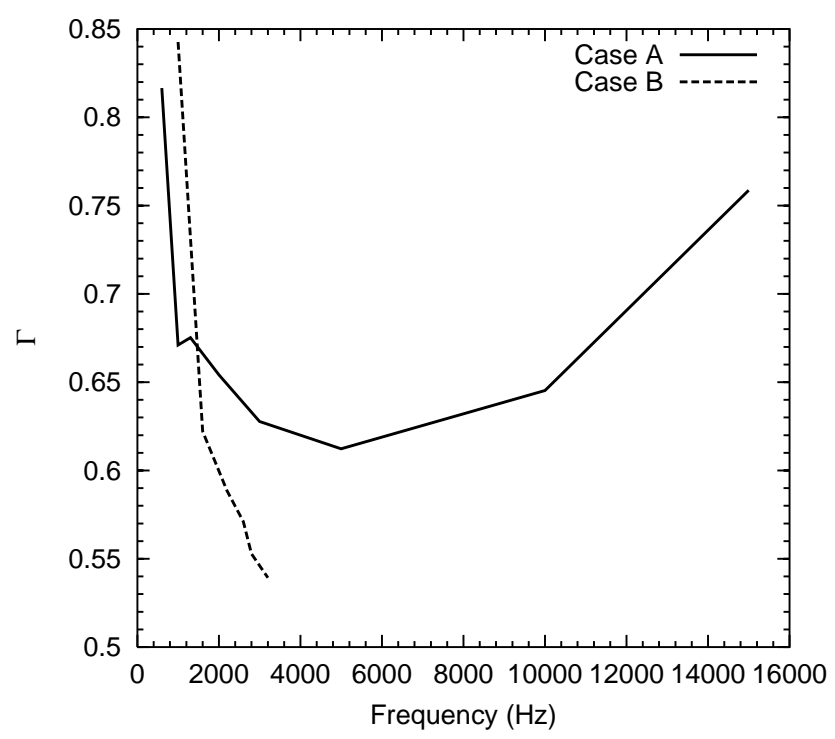

Figure 12. Ignition parameter as a function of frequency for cases A and B 
of $\chi$ cycle lies in favorable region for ignition since $\chi_{a v}>\chi_{s}$. This reduces $\Gamma$ as frequency increases. It is very interesting to see that the curves for different values of $V_{0}$ and $A$ collapse very well. Note that all the chosen values of $V_{0}$ are very close to the steady ignition limit and steady ignition delay is highly sensitive to $V_{0}$ in that region (see Fig. 1), still this map of ignition delay response to $\Gamma$ is unique for all the chosen parameters. Therefore, this curve uniquely describes the ignition delay response to frequency for different amplitudes and $V_{0}$ of velocity oscillation at the nozzle, for all cases which have $\chi_{a v}>\chi_{s}$.

Another important thing to note is that all the curves bend up sharply at a value of $\Gamma \approx 0.55$. It is found that higher frequencies that do not ignite have $\Gamma$ value less than 0.55 . Therefore, it is appropriate to define an ignitability criterion based on $\Gamma$ in the sense that $\Gamma$ must be greater than 0.55 for ignition to occur.

To further explain the differences in ignition response for cases $A$ and $B$, we plot $\Gamma$ as a function of frequency for cases A and B in Fig. 12. We can see that for case B $\Gamma$ continues to decrease as frequency increases, and reaches a cut-off value of 0.55 , after which no ignition takes place. For case A, however, $\Gamma$ decreases initially and then increases. Since it never becomes less than 0.55 for case A, all the frequencies for case A ignite.

\section{Conclusions}

The effects of unsteady scalar dissipation rate on ignition of hydrogen/air mixing layer are studied using counterflow configuration. Axial velocity at the nozzle inlet is imposed as a sinusoidal function in time, and the corresponding variation in the scalar dissipation rate at the ignition kernel is measured as the main parameter of the study. Two cases are studied: $\chi_{a v}<\chi_{s}$ and $\chi_{a v}>\chi_{s}$. While both cases show similar ignition delay response to low frequencies of velocity oscillation, at high frequencies the ignition delay response is significantly different for the two cases. Low frequency ignition delay response is quasi-steady and correlates well with the mean scalar dissipation rate for both the cases. In the high frequency regime, for the case with $\chi_{a v}<\chi_{s}$, ignition delay increases with frequency, and then levels off at higher frequencies. On the other hand, for $\chi_{a v}>\chi_{s}$, ignition delay increases monotonically with frequency up to $2800 \mathrm{~Hz}$, beyond which no ignition is observed. For both cases, ignition delay behavior is explained by introducing a new ignition parameter $(\Gamma)$ based on Damköhler number of $\mathrm{H}$ radicals, computed at the ignition kernel. $\Gamma$ is basically the fractional duration of favorable condition for ignition. Thus ignition delay is found to increase with a reduction in $\Gamma$ for both the cases. Various other cases for different values of $V_{0}$ and $A$ are considered with $\chi_{a v}>\chi_{s}$, and their ignition response to frequency is found to collapse into a single curve using this newly proposed ignition parameter. The criterion for non-ignitability is also proposed based on the same ignition parameter. Critical value of ignition parameter is found to be 0.55 , below which the mixture does not ignite. Thus, the study presents a unified way to characterize the ignition delay response to scalar dissipation rate fluctuations.

\section{Acknowledgments}

This study was supported by the University Consortium on Low Temperature Combustion for HighEfficiency, Ultra-Low Emission Engines directed by the University of Michigan and funded by the Department of Energy. SRL would like to thank the financial support by Seoul National University of Technology.

\section{References}

\footnotetext{
${ }^{1}$ D. Thevenin and S. Candel, Proc. Combust. Inst. 25 (1994) 1547-1554.

${ }^{2}$ G. Balakrishnan, M.D. Smooke, F.A. Williams, Combust. Flame 102 (1995) 329-340.

${ }^{3}$ A.L. Sánchez, G. Balakrishnan, A. Liñán, and F.A. Williams, Combust. Flame 105 (1996) 569-590.

${ }^{4}$ C.G. Fotache, T.G. Kreutz, and C.K. Law, Combust. Flame 110 (1997) 429-440.

${ }^{5}$ T. G. Kreutz and C. K. Law, Combust. Flame 104 (1996) 157-175.

${ }^{6}$ C.J. Sung and C.K. Law, Combust. Sci. Technol. 129 (1997) 347-370.

${ }^{7}$ H.G. Im, L.L. Raja, R.J. Kee, and L.R. Petzold, Combust. Sci. Technol. 158 (2000) 341-363.

${ }^{8}$ S.D. Mason, J.H. Chen and H.G. Im, Proc. Combust. Inst. 29 (2002) 1629-1636.

${ }^{9}$ G. Bansal, H.G. Im and S.R. Lee, Combust. Theory Modelling, submitted (2008).

${ }^{10}$ H.G. Im, L.L. Raja, R.J. Kee, A.E. Lutz, and L.R. Petzold, OPUS: A Fortran Program for Unsteady Opposed-Flowed Flames, Report No. SAND2000-8211, Sandia National Laboratories, 2000.

${ }^{11} \mathrm{~S}$. Li and L.R. Petzold, Design of New DASPK for Sensitivity Analysis, Report No. TRCS99-23, Technical report of computer science department, University of California, Santa Barbara, 1999.
} 
${ }^{12}$ R.J. Kee, F.M. Rupley, and J.A. Miller, Chemkin-II, A Fortran Chemical Kinetics Package for the Analysis of Gas-Phase Chemical Kinetics, Report No. SAND89-8009B, Sandia National Laboratories, 1991.

${ }^{13}$ R.J. Kee, G. Dixon-Lewis, J. Warnatz, M.E. Coltrin, and J.A. Miller, A Fortran Computer Code Package for the Evaluation of Gas-Phase Multicomponent Transport Properties, Report No. SAND86-8246, Sandia National Laboratories, 1986.

${ }^{14}$ R.A. Yetter, F.L. Dryer, and H. Rabitz, Combust. Sci. Technol. 79 (1991) 97-128.

${ }^{15}$ C. K. Law, Combustion physics, Cambridge University Press (2006).

${ }^{16}$ R.W. Bilger, Proc. Combust. Inst. 22 (1988) 475-488. 